\title{
The Myristylation Motif of Pto Is Not Required for Disease Resistance
}

\author{
Ying-Tsu Loh, Jianmin Zhou, and Gregory B. Martin \\ Department of Agronomy, 1150 Lilly Hall of Life Sciences, Purdue University, West Lafayette, IN 47907- \\ 1150, U.S.A. \\ Accepted 14 February 1998.
}

\begin{abstract}
The tomato Pto kinase confers resistance to bacterial speck disease caused by strains of Pseudomonas syringae pv. tomato that express the avirulence gene avrPto. Pto contains a putative myristylation site at its amino terminus that was hypothesized to play a role in localizing Pto in the plant cell. Site-directed mutagenesis was used to change the invariant glycine residue in the myristylation motif to an alanine. Transgenes encoding the mutant Pto(G2A) and wild-type Pto were placed behind the cauliflower mosaic virus $35 \mathrm{~S}$ promoter and transformed into tomato plants that are susceptible to bacterial speck disease. Both the mutant and wild-type forms of Pto conferred resistance to a strain of $P$. syringae pv. tomato expressing avrPto. These results indicate that the myristylation motif of Pto is not required for bacterial speck disease resistance.
\end{abstract}

Additional keywords: Fen, signal transduction, tyrosine kinase.

The Pto gene was isolated by map-based cloning and encodes a serine/threonine protein kinase that confers resistance to strains of Pseudomonas expressing the avirulence gene avrPto (Martin et al. 1993; Loh and Martin 1995). In this gene-for-gene interaction, the Pto kinase has roles in both recognition of the pathogen and in signal transduction leading to the activation of plant defense responses. For example, it was recently shown that the Pto kinase physically interacts with the AvrPto protein and that this recognition event initiates disease resistance (Scofield et al. 1996; Tang et al. 1996). Furthermore, Pto associates with several Pto-interacting (Pti) proteins that appear to participate in distinct signaling pathways. Pti1 is a serine-threonine kinase that is phosphorylated by Pto and probably acts downstream in a pathway leading to the hypersensitive response (Zhou et al. 1995). The Pti4, Pti5, and Pti6 proteins are putative transcription factors that bind to a cis element in the promoters of many pathogenesis-related genes (Zhou et al. 1997). Pto-mediated resistance also requires Prf, which encodes a protein containing a leucine-rich

Corresponding author: G. B. Martin; Telephone: 765-494-4790; Fax: 765-496-2926; E-mail: gmartin@ dept.agry.purdue.edu

Present address of Jianmin Zhou: Department of Plant Pathology, 4024 Throckmorton Plant Sciences Center, Kansas State University, Manhat$\tan 66506-5502$, U.S.A. region and a putative nucleotide binding site, although the role of this protein remains unclear (Salmeron et al. 1996).

The subcellular localization of the Pto kinase is unknown. However, a region in the amino terminus of Pto contains a sequence (MGSKYSK) that shares similarity with a consensus myristylation motif (MG[SVALQN]xx[STAGCN] not P\}; Towler et al. 1988). N-myristylation is catalyzed by myristyl CoA:protein N-myristyltransferase and involves the cotranslational amide linkage of myristic acid (C14:0) to a glycine residue penultimate to the $\mathrm{N}$ terminus (Wilcox et al. 1987; Deichaite et al. 1988). Myristylation plays important roles both in the subcellular localization of proteins by targeting them to cellular membranes and in the stabilization of protein structure (Resh 1990; Kataoka et al. 1993). The role of protein $\mathrm{N}$-myristylation has received little attention in plants although it has been examined in several mammals (Boutin 1997).

Myristylated proteins in mammals are often involved in signal transduction and include the tyrosine kinase, p60 ${ }^{v-s r c}$, and src family members src, lyn, and lck, the cyclic AMPdependent protein kinase catalytic subunit, and the calcineurin B-subunit protein phosphatase (Towler et al. 1988; Bhatnagar and Gordon 1997; Boutin 1997). The requirement of myristylation for function of $\mathrm{p} 60^{v-s r c}$ was demonstrated by mutagenesis of the N-terminal glycine residue of the myristylation motif. This mutation resulted in loss of the ability of $\mathrm{p} 60^{v-s r c}$ to associate with cellular membranes and to transform chicken embryo cells (Buss et al. 1986). In another study, the addition of a myristylation motif to the Akt serine/threonine kinase caused it to localize to the plasma membrane, where it was constitutively activated by several receptor tyrosine kinases (Kohn et al. 1996; Hemmings 1997; Franke et al. 1997). However, not all proteins that contain a myristylation motif are myristylated and some proteins that are myristylated clearly do not require it for their function (Resh 1994; Zhu et al. 1995).

Myristylation has not been well characterized in plants although a gene encoding myristyl CoA:protein N-myristyltransferase has been isolated from Arabidopsis thaliana (GenBank accession no. T21207). In addition to the Pto kinase, several other plant protein kinases have been observed to have putative myristylation sites, including the closely related Fen kinase from tomato, the ATN1 and APK1 kinases from A. thaliana, and several calcium-dependent kinases from 
maize (Hirayama and Oka 1992; Martin, et al. 1994; Tregear et al. 1996; Saijo et al. 1997). A myristylation motif is also present in the derived amino acid sequences from several cDNAs encoding fatty acyl-Co-A synthetase from Brassica napus and from a nodule-specific gene family from Alnus glutinosa (Fulda et al. 1997; Pawlowski et al. 1997). It has not been reported whether the myristylation motifs in any of these proteins are required for their function. In this report we describe a mutational analysis of the myristylation motif of Pto and show that it is not required for the disease resistance conferred by this protein.

The myristylation motif of Pto was found by a MOTIFS search (University of Wisconsin GCG package) for amino acid sequences that have a role in protein function (Fig. 1A). Although the consensus myristylation motif spans seven amino acids, the only absolute requirements for N-myristylation have been shown to be a glycine adjacent to the initial methionine and, for a few proteins, a serine/threonine five residues from the initial methionine (Boutin 1997). The myristylation motif of Pto contains these critical residues and is overall highly similar to the myristylation site found in the p60 ${ }^{v-s r c}$ tyrosine kinase (Martin et al. 1993; Fig. 1A).

To examine the possible requirement of the myristylation motif for Pto-mediated disease resistance, we developed transgenic plants expressing a mutant version of Pto. The myristylation motif was mutated by changing the invariant glycine residue to an alanine residue by polymerase chain reaction (PCR) site-directed mutagenesis (Fig. 1B). A glycineto-alanine substitution has been shown to abolish myristylation of the p60 $0^{v-s r c}$ tyrosine kinase (Buss et al. 1986). Briefly, primers JZ10 (5'-CTCAAGCTTAATCATGGCAAGCAAGT ATTCTAAGG-3') and YTL5 (5'-CCCTGCAGTGAAAGAA GGATCCACAG-3') were used to amplify a 1,055-base pair (bp) product encoding Pto from plasmid PTC3 (PTC3 is CD186 in plasmid pCDNAII [Invitrogen, San Diego, CA]) (Martin et al. 1993). Primer JZ10 introduced the nucleotide change GCA (coding for alanine) in place of GGA (coding for glycine) at the $5^{\prime}$ end of the Pto gene and also created a HindIII restriction site 9 bp upstream of the ATG start codon. Primer YTL5 amplified a BamHI restriction site $70 \mathrm{bp}$ after the TAA stop codon. The PCR product was digested with HindIII and BamHI to obtain a 1,042-bp fragment that was inserted into vector pGEM-7Zf(+), which had been digested with the corresponding enzymes. The resulting mutated gene, 46-Pto ${ }^{G 2 A}$, was confirmed by sequencing the entire gene.

Two other Pto constructs were also made by PCR amplification, including an HA (hemaglutinin) epitope-tagged mutated Pto (47-Pto $\left.{ }^{G 2 A}-H A\right)$, and an HA-tagged wild-type Pto (48-Pto-HA). The HA tag was added for future experiments aimed at localizing the Pto protein in plant cells. The wildtype Pto-HA construct was made with the primers T7 (5'AATACGACTCACTATAG-3') and JZ9 (5'AAAGAGCTCA AGCGTAATCTGGAACATCGTATGGGTAAATAACAGAC TCTTGGAGACG-3'). Primer JZ9 introduced a 27-bp sequence encoding the HA epitope and a SacI site at the $3^{\prime}$ end of Pto, whereas T7 allowed amplification from the vector sequence at the $5^{\prime}$ end. This amplified product was digested with EcoRI and SacI to obtain a 1,058-bp segment that was inserted into vector pGEM-7Zf(+), which had been digested with the corresponding enzymes. The correct insertion of the HA tag was confirmed by sequencing the entire altered Pto gene. To make the Pto ${ }^{G 2 A}-H A$ gene, 46-Pto ${ }^{G 2 A}$ was digested with $X b a \mathrm{I} / B g l \mathrm{II}$ and the 450 -bp fragment containing a portion of the Pto open reading frame was isolated (Fig. 1B). This fragment was then inserted into the same region of the 48-Pto$H A$ construct to obtain $47-P t o^{G 2 A}-H A$. Again, the entire mutated $P$ to gene was confirmed by sequencing.

To produce constructs for plant transformation, the Pto gene was removed from each pGEM-7Zf(+)-based plasmid by digestion with $X b a \mathrm{I} / S a c \mathrm{I}$. The $X b a \mathrm{I} / S a c \mathrm{I}$ fragments were ligated into plasmid pBI121 in the sense orientation under the control of the cauliflower mosaic virus (CaMV) 35S promoter, and
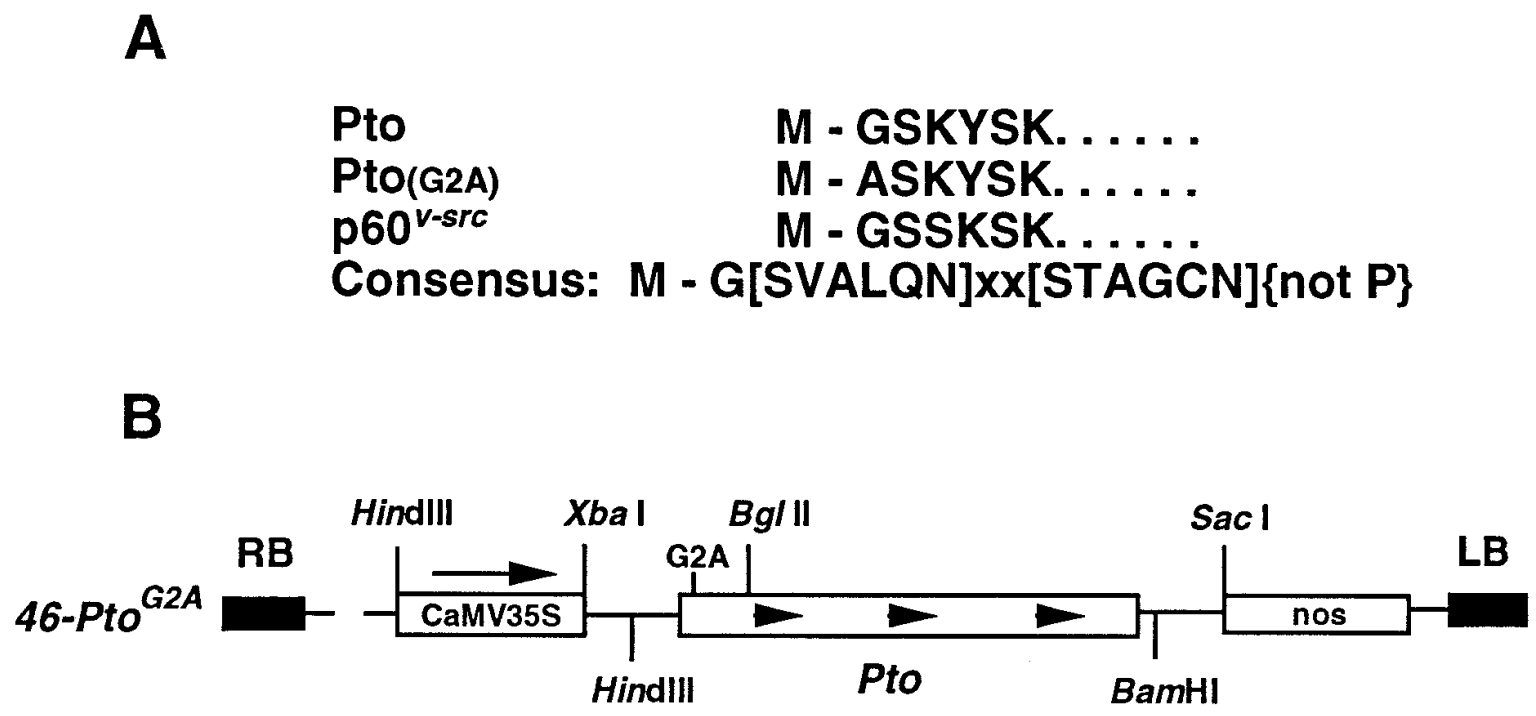

Fig. 1. The Pto myristylation motif and the Pto transgenes. A, Comparison of the Pto myristylation motif with that of the p60 $0^{v-s r c}$ tyrosine kinase and a consensus myristylation site derived from various proteins (Boutin 1997; Towler et al. 1988). B, Physical map of the 46-Pto ${ }^{2 A}$ transgene that was introduced into bacterial speck-susceptible Moneymaker tomato plants. Construct 46-Pto ${ }^{G 2 A}$ contains the glycine-to-alanine residue change in the second position of the Pto amino acid sequence. Transgene 47-Pto ${ }^{G 2 A}-H A$ is the same as $46-P t o^{G 2 A}$ except for the presence of a $27-$ base pair hemaglutinin (HA) epitope tag at the carboxyl terminus (see text). Transgene 48-Pto-HA is wild-type Pto with the HA tag at the carboxyl terminus (see text). 
the resulting construct was electroporated into Agrobacterium strain EHA105 (Fig. 1B). Each transgene was introduced into the bacterial speck susceptible tomato line Moneymaker by Agrobacterium-mediated transformation (McCormick et al. 1986).

Putative transformed tomato lines were identified for each of the transgenes by selection on kanamycin-containing medium. To confirm the transformants, we isolated genomic DNA from each original transformant $\left(\mathrm{R}_{1}\right)$ and from at least 12 progeny of each $\mathrm{R}_{1}$ plant, digested the DNAs with PstI and $B g l$ II restriction enzymes, and analyzed them on DNA gel blots with a radiolabeled Pto gene probe (Fig. 2). BglII cuts within the transgene, whereas PstI was chosen because it does not cut within the transgene and allows identification of different integration events. This analysis showed that independent transformants had been obtained for the 46-Pto ${ }^{G 2 A}$ transgene ( $\mathrm{R}_{1}$ plants 46-1, 46-3, and 46-6), the 47-Pto ${ }^{G 2 A}-H A$ transgene $\left(\mathrm{R}_{1}\right.$ plants $47-1$ and 47-14), and the 48-Pto-HA transgene ( $\mathrm{R}_{1}$ plant $\left.48-2\right)$ (Fig. 2$)$. A few plants contained several new restriction fragments that hybridized to Pto, indicat-

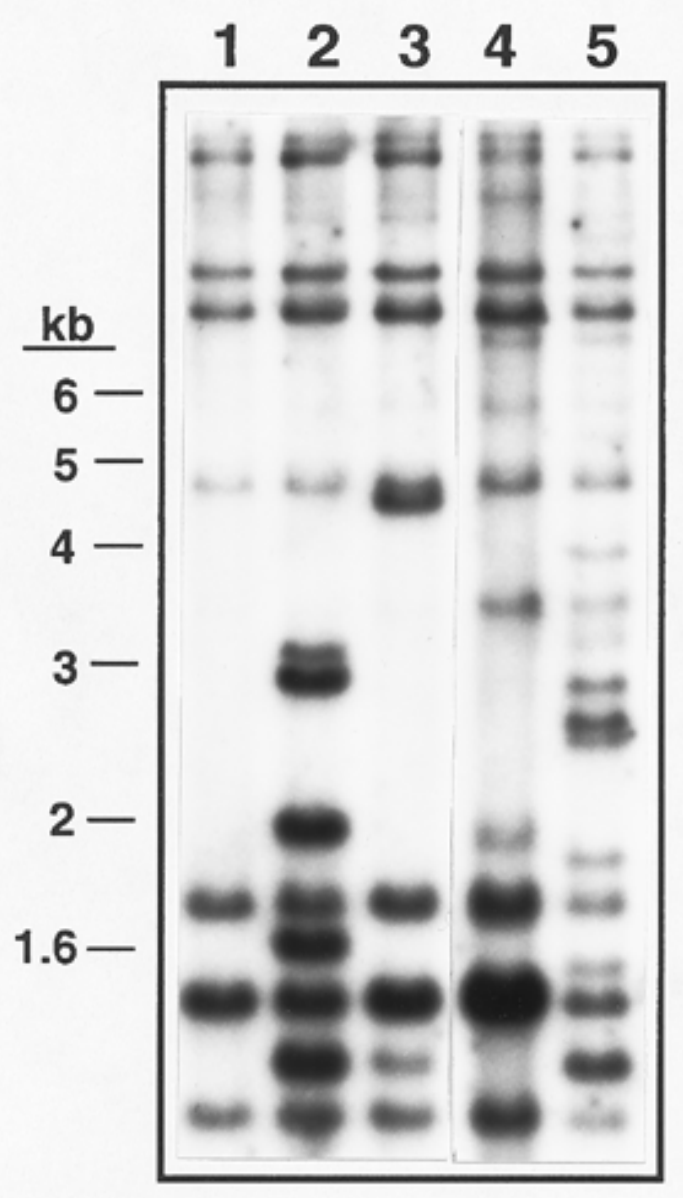

Fig. 2. DNA gel blot analysis of representative transgenic plants. Approximately $5 \mu \mathrm{g}$ of tomato genomic DNA was digested with PstI and $B g l \mathrm{II}$, separated on a $1 \%$ agarose gel, and blotted onto Hybond N+ membrane. The blot was probed with a radiolabeled Pto gene probe. Lane 1 contains DNA from a nontransgenic Moneymaker plant. Lanes 2 to 5 contain DNAs from resistant progeny derived from original transformants 46-1, 46-3, 47-1, and 47-14, respectively. Progeny from original transformants $46-6$ and $48-2$ were also found to contain new fragments hybridizing to the Pto probe (data not shown). ing they may contain multiple copies of the transgene (Fig. 2). Each original $\left(\mathrm{R}_{1}\right)$ plant was allowed to self-fertilize, and the seeds were collected for analysis of the $\mathrm{R}_{2}$ progeny.

To confirm that the transformed plants contained the correct transgenes, we amplified the region encompassing the introduced Pto gene from two representative plants, 46-3 (carrying transgene 46-Pto ${ }^{G 2 A}$ ) and 48-2 (carrying transgene 48-Pto-HA), using primers targeted to the CaMV $35 \mathrm{~S}$ promoter and the nos termination sequence (see Figure 1B), and subcloned the products into vector pCRII (Invitrogen). The insert from one clone derived from each line was sequenced (Fig. 3). The expected nucleotide change was observed at the codon encoding the glycine residue (now an alanine) in the Pto myristylation motif from 46-Pto ${ }^{G 2 A}$ transgenic plant and the wild-type sequence was present in the 48-Pto-HA plant (Fig. 3).

We next tested the requirement of the myristylation motif for the function of the Pto kinase in conferring resistance to a Pseudomonas syringae pv. tomato (Pst) strain expressing avrPto. Ten-week-old $\mathrm{R}_{2}$ progeny derived from each of the original transformants were inoculated by dipping leaves in bacterial suspensions of a virulent Pst strain (T1) lacking avrPto and an avirulent Pst strain expressing avrPto [T1(avrPto)] (Table 1). As controls, resistant cultivar Rio Grande-PtoR (Pto/Pto) and susceptible cultivar Moneymaker (pto/pto) were also inoculated. Bacteria were grown overnight in liquid King's B Medium with selection (100 $\mu \mathrm{g}$ of rifampicin per $\mathrm{ml}$ for $\mathrm{T} 1$ and $100 \mu \mathrm{g}$ of rifampicin per $\mathrm{ml}$ and $25 \mu \mathrm{g}$ of kanamycin per $\mathrm{ml}$ for T1[avrPto]). Cells were pelleted, resuspended, and washed twice with $10 \mathrm{mM} \mathrm{MgCl}_{2}$. A final suspension of $2 \times 10^{7} \mathrm{CFU} / \mathrm{ml}$ was prepared in $10 \mathrm{mM} \mathrm{MgCl}_{2}$ with $0.05 \%$ Silwet. Separate leaves of each plant were dipped in $\mathrm{T} 1$ or T1(avrPto), and plants were scored for symptoms of bacterial speck disease 5 days after inoculation.

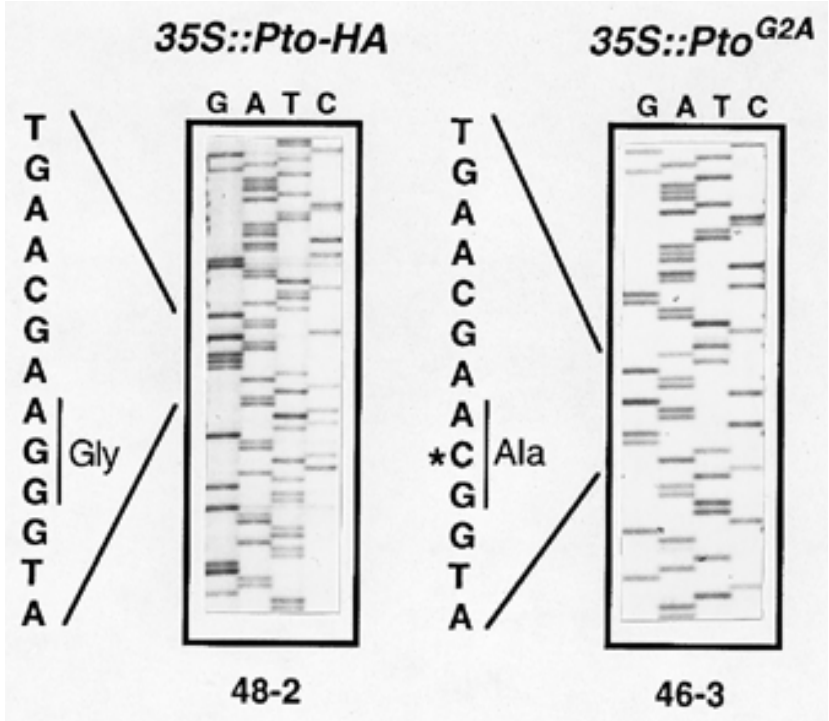

Fig. 3. DNA sequence of the transgene isolated from a resistant progeny plant derived from $\mathrm{R}_{1}$ plant $46-3$ (Pto ${ }^{G 2 A}$ ) or $48-2$ (Pto-HA). The Pto transgene was amplified from genomic DNA with primers targeted to the cauliflower mosaic virus $35 \mathrm{~S}$ promoter and the nos termination sequence. The amplified product was cloned into pCRII vector and sequenced with M13R and M13F primers. The mutated nucleotide in the second codon of Pto in plant 46-3 is indicated with an asterisk. 
Rio Grande-PtoR (Pto/Pto) developed no symptoms of bacterial speck after inoculation with avirulent T1(avrPto), whereas Moneymaker (pto/pto) developed typical necrotic lesions surrounded by chlorotic halos (not shown). All plants that were inoculated with the virulent $P$. syringae pv. tomato strain T1 lacking avrPto developed typical symptoms of bacterial speck disease. Progeny from each of the original transformants, and notably the Pto ${ }^{G 2 A}$ transformants, segregated in a 3:1 ratio (resistant:susceptible) consistent with the ability of the Pto transgenes to confer avrPto-specific resistance (Table 1). All of the progeny that expressed resistance to T1(avrPto) contained a Pto transgene as determined by DNA gel blots, whereas none of the susceptible progeny contained a Pto transgene (data not shown). Chi-square analysis indicated that the segregation ratios are consistent with there being a single integration event of at least one active Pto transgene in each of the original transformants (Table 1). This conclusion is further supported by perfect co-segregation of all new restriction fragments that hybridized to the radiolabeled Pto probe (data not shown). Based on this genetic analysis, we conclude that the myristylation motif of the Pto kinase is not necessary for its function in conferring resistance to $P$. syringae pv. tomato expressing avrPto.

For this study, we used the CaMV 35S promoter and not the native Pto promoter to express the transgenes. It is possible, therefore, that increased abundance of the Pto kinases affected their localization. However, two observations indicate that the abundance of the kinases was not greatly increased. First, RNA blot analysis indicated that, in common with wild-type plants, Pto transcripts are present in very low abundance in the transgenic plants. This may be due to possible deleterious effects of Pto overexpression. Secondly, using an antibody prepared against a synthetic peptide corresponding to a highly divergent region of the Pto kinase, we have been unable to detect an increase in Pto protein in the transgenic plants (data not shown).

In a previous study, the function of the myristylation motif in the Fen kinase was tested with a gene expression system based upon potato virus X (PVX) (Rommens et al. 1995). In that work, the nucleotide sequence coding for the invariant glycine residue was altered to a sequence coding for arginine, cloned into PVX, and transiently expressed in fenthioninsensitive tomato plants. The tomato plants showed no sensitivity to the insecticide, and it was concluded that the myristylation motif is necessary for Fen to mediate fenthion sensitivity. The lack of fenthion sensitivity in plants infected with PVX::Fen $(G 2 R)$ could have several interpretations. It may result from instability of the Fen protein caused by sub- stitution of an arginine residue for the invariant glycine or from possible instability of the viral genome that is sometimes observed with the PVX system (Chapman et al. 1992; Rommens et al. 1995). Alternatively, the different results seen with the Pto and Fen myristylation mutants may represent a fundamental difference in how the two kinases are localized and function in the plant cell.

We have shown that a site-directed mutation of the invariant glycine in the Pto myristylation motif does not affect the ability of the kinase to confer avrPto-specific resistance to bacterial speck disease. This result is consistent with our finding that interference with the myristylation motif by formation of carboxyl terminus fusions with LexA and MBP does not affect the physical interaction of Pto with AvrPto or with Ptil in the yeast two-hybrid system nor does it affect in vitro kinase activity of Pto (Tang et al. 1996; Zhou et al. 1995; Loh and Martin 1995). Finally, this work has demonstrated that an epitope tag inserted at the C-terminal end of the Pto kinase does not affect its function in disease resistance. The epitope tag should prove useful for in vivo studies of Pto, which previously have been hindered by the presence of several Pto homologous proteins in tomato.

\section{ACKNOWLEDGMENTS}

We thank Roger Thilmony for helpful discussions and inoculation of plants and Leslie Lincoln for technical assistance in the isolation of genomic DNA and the screening of plants. For critical reading of the manuscript we thank Larry Dunkle, Yong Gu, Marietta Harrison, Roger Thilmony, and Jeff Volenec. This research was supported by National Science Foundation grant MCB-96-30635 (G. B. M.) and a David and Lucille Packard Foundation Fellowship (G. B. M.).

\section{LITERATURE CITED}

Bhatnagar, R. S., and Gordon, J. I. 1997. Understanding covalent modifications of proteins by lipids: Where cell biology and biophysics mingle. Trends Cell Biol. 7:14-20.

Boutin, J. A. 1997. Myristylation. Cell. Signalling 9:15-35.

Buss, J. E., Kamps, M. P., Gould, K., and Sefton, B. M. 1986. The absence of myristic acid decreases membrane binding of $\mathrm{p} 60^{\text {src }}$ but does not affect tyrosine protein kinase activity. J. Virol. 58:468-474.

Chapman, S., Kavanagh, T., and Baulcombe, D. 1992. Potato virus X as a vector for gene expression in plants. Plant J. 2:549-557.

Deichaite, I., Casson, L. P., Ling, H.-P., and Resh, M. D. 1988. In vitro synthesis of pp60 ${ }^{v-s r c}$ : Myristylation in a cell-free system. Mol. Cell. Biol. 8:4295-4301.

Franke, T. F., Kaplan, D. R., Cantley, L. C., and Toker, A. 1997. Direct regulation of the Akt proto-oncogene product by phsophatidylinositol-3,4-bisphosphate. Science 275:665-668.

Fulda, M., Heinz, E., and Wolter, F. P. 1997. Brassica napus cDNAs encoding fatty acyl-CoA synthetase. Plant Mol. Biol. 33:911-922.

Table 1. Genetic analysis of Pto transgenic plants for reaction to Pseudomonas syringae pv. tomato (avrPto $)^{\mathrm{a}}$

\begin{tabular}{|c|c|c|c|c|c|c|c|}
\hline \multirow[b]{2}{*}{$R_{1}$ plant } & \multirow[b]{2}{*}{ Genotype } & \multicolumn{3}{|c|}{$\mathbf{R}_{2}$ progeny } & \multirow[b]{2}{*}{$\chi^{2}$} & \multirow[b]{2}{*}{ Segregation ratio tested } & \multirow[b]{2}{*}{$\mathbf{P}$} \\
\hline & & Resistant & Susceptible & Total & & & \\
\hline $46-1$ & $35 S: \because$ Pto $^{G 2 A}$ & 10 & 5 & 15 & 0.5555 & $3: 1$ & 0.46 \\
\hline $46-3$ & $35 S::$ Pto $^{G 2 A}$ & 10 & 4 & 14 & 0.0952 & $3: 1$ & 0.76 \\
\hline $46-6$ & $35 S::$ Pto $^{G 2 A}$ & 13 & 3 & 16 & 0.3333 & $3: 1$ & 0.56 \\
\hline $47-1$ & $35 \mathrm{~S}: \because \mathrm{Pto}^{G 2 A}-\mathrm{HA}$ & 9 & 1 & 10 & 1.2000 & $3: 1$ & 0.27 \\
\hline $47-14$ & $35 S: \because$ Pto $^{G 2 A}-H A$ & 8 & 3 & 11 & 0.0303 & $3: 1$ & 0.86 \\
\hline $48-2$ & 35S::Pto-HA & 9 & 2 & 11 & 0.2727 & $3: 1$ & 0.60 \\
\hline
\end{tabular}

${ }^{a}$ Leaves of each plant were inoculated with a suspension of $2 \times 10^{7} \mathrm{CFU} / \mathrm{ml}$ of $P$. syringae pv. tomato strain T1 expressing avrPto. Leaves were scored for symptoms of bacterial speck disease 5 days after inoculation. 
Hemmings, B. A. 1997. Akt signaling: Linking membrane events to life and death decisions. Science 275:628-630.

Hirayama, T., and Oka, A. 1992. Novel protein kinase of Arabidopsis thaliana (APK1) that phosphorylates tyrosine, serine and threonine. Plant Mol. Biol. 20:653-662.

Kataoka, M., Mihara, K., and Tokunaga, F. 1993. Recoverin alters its surface properties depending on both calcium-binding and $\mathrm{N}$-terminal myristylation. J. Biochem. 114:535-540.

Kohn, A. D., Takeuchi, F., and Roth, R. A. 1996. Akt, a pleckstrin homology domain containing kinase, is activated primarily by phosphorylation. J. Biol. Chem. 271:21920-21926.

Loh, Y.-T. and Martin, G. B. 1995. The Pto bacterial resistance gene and the Fen insecticide sensitivity gene encode functional protein kinases with serine/threonine specificity. Plant Physiol. 108:17351739.

Martin, G. B., Brommonschenkel, S. H., Chunwongse, J., Frary, A., Ganal, M. W., Spivey, R., Wu, T., Earle, E. D., and Tanksley, S. D. 1993. Map-based cloning of a protein kinase gene conferring disease resistance in tomato. Science 262:1432-1436.

Martin, G. B., Frary, A., Wu, T., Brommonschenkel, S., Chunwongse, J., Earle, E. D., and Tanksley, S. D. 1994. A member of the Pto gene family confers sensitivity to fenthion resulting in rapid cell death. Plant Cell 6:1543-1552.

McCormick, S., Niedermeyer, J., Fry, J., Barnason, A., Horsch, R., and Fraley, R. 1986. Leaf disc transformation of cultivated tomato (L. esculentum) using Agrobacterium tumefaciens. Plant Cell Rep. 5:81-84.

Pawlowski, K., Twigg, P., Dobritsa, S., Guan, C., and Mullin, B. C 1997. A nodule-specific gene family from Alnus glutinosa encodes glycine- and histidine-rich proteins expressed in the early stages of actinorhizal nodule development. Mol. Plant-Microbe Interact. 10: 656-664.

Resh, M. D. 1990. Membrane interactions of pp60v-src: A model for myristylated tyrosine protein kinases. Oncogene 5:1437-1444.

Resh, M. D. 1994. Myristylation and palmitylation of Src family members: The fats of the matter. Cell 76:411-413.

Rommens, C. M. T., Salmeron, J. M., Baulcombe, D. C., and Staskawicz, B. J. 1995. Use of a gene expression system based on potato virus $\mathrm{X}$ to rapidly identify and characterize a tomato Pto homolog that controls fenthion sensitivity. Plant Cell 7:249-257.

Saijo, Y., Hata, S., Sheen, J., and Izui, K. 1997. cDNA cloning and prokaryotic expression of maize calcium-dependent protein kinases. Biochim. Biophys. Acta 1350:109-114.

Salmeron, J. M., Oldroyd, G. E. D., Rommens, C. M. T., Scofield, S. R., Kim, H.-S., Lavelle, D. T., Dahlbeck, D., and Staskawicz, B. J. 1996. Tomato Prf is a member of the leucine-rich repeat class of plant disease resistance genes and lies embedded within the Pto kinase gene cluster. Cell 86:123-133.

Scofield, S. R., Tobias, C. M., Rathjen, J. P., Chang, J. H., Lavelle, D. T. Michelmore, R. W., and Staskawicz, B. J. 1996. Molecular basis of gene-for-gene specificity in bacterial speck disease of tomato. Science 274:2063-2065.

Tang, X., Frederick, R. D., Zhou, J., Halterman, D. A., Jia, Y., and Martin, G. B. 1996. Initiation of plant disease resistance by physical interaction of AvrPto and Pto kinase. Science 274:2060-2063.

Towler, D. A., Gordon, J. I., Adams, S. P., and Glaser, L. 1988. The biology and enzymology of eukaryotic protein acylation. Annu. Rev. Biochem. 57:69-99.

Tregear, J. W., Jouannic, S., Schwebel-Dugue, N., and Kreis, M. 1996. An unusual protein kinase displaying characteristics of both the serine/threonine and tyrosine families is encoded by the Arabidopsis thaliana gene ATN1. Plant Sci. 117:107-119.

Wilcox, C., Hu, J.-S., and Olson, E. N. 1987. Acylation of proteins with myristic acid occurs co-translationally. Science 238:1275-1278.

Zhou, J., Loh, Y.-T., Bressan, R. A., and Martin, G. B. 1995. The tomato gene Ptil encodes a serine/threonine kinase that is phosphorylated by Pto and is involved in the hypersensitive response. Cell 83:925-935.

Zhou, J., Tang, X., and Martin, G. B. 1997. The Pto kinase conferring resistance to tomato bacterial speck disease interacts with proteins that bind a cis-element of pathogenesis-related genes. EMBO J. 16: 3207-3218.

Zhu, D., Cardenas, M. E., and Heitman, J. 1995. Myristoylation of calcineurin $\mathrm{B}$ is not required for function or interaction with immunophilin-immunosuppresant complexes in yeast Saccharomyces cerevisae. J. Biol. Chem. 270:24831-24838. 